\title{
Intercostal nerve conduction study in man
}

\author{
SUNIL PRADHAN, * ARUN TALY \\ From the Department of Neurology, National Institute of Mental Health and Neurosciences, Bangalore, India
}

SUMMARY A new surface technique for the conduction study of the lower intercostal nerves has been developed and applied to 30 normal subjects. The problem of the short available nerve segment of the intercostal nerves and the bizzare compound motor action potential (CMAP) of inconsistent latency while recording over the intercostal muscles, is overcome by applying recording electrodes over the rectus abdominis muscle and stimulating the nerves at two points at a fair distance away. With the use of multiple recording sites over the rectus abdominis, the motor points for different intercostal nerves were delineated. CMAP of reproducible latencies and waveforms with sharp take-off points were obtained. Conduction velocity of the intercostal nerves could be determined.

There is no standard electrophysiological method of studying the nerves of the trunk in man. Even for the neuropathies which preferentially involve the truncal nerves, for example diabetic thoraco-abdominal radiculoneuropathy ${ }^{1-3}$ and segmental zoster paralysis, ${ }^{45}$ electrophysiological studies have been confined to nerve conduction in the extremities and concentric needle electromyography of paraspinal and abdominal muscles. The basic physiological studies of intercostal nerves have also been confined to concentric needle electromyography of the intercostal muscles. ${ }^{6-8}$ The present study of intercostal nerve conduction is to our knowledge the first of this kind. It is aimed at finding out the proper stimulating and recording sites for different intercostal nerves so as to obtain consistent results. The technique was standardised accordingly and its reliability judged by observing the reproducibility of results obtained at different time intervals in the same individual.

\section{Methods}

Thirty normal volunteers with no evidence of peripheral neuropathy, pulmonary disease, chest deformity, abdominal operation or injury were the subjects for this study. Subjects were asked to lie on their side opposite to the side of examination. The arms were rested over the head. Intercostal spaces were made wide and prominent by putting a pillow under the chest. Subjects were not allowed to change their position during the period of study. Total relaxation and

\footnotetext{
* Present address and address for reprint requests: Dr Sunil Pradhan, Department of Neurology, Nizam's Institute of Medical Sciences, Punjagutta, Hyderabad-500 482, India.
}

Received 21 October 1988.

Accepted 28 November 1988 quiet breathing were assured by prior explanation of the procedure. Holding of breath was not found necessary for the study. A comma shaped stimulator was placed in the intercostal spaces with the cathode $3 \mathrm{~cm}$ anterior to the anode. It was gently pressed deep and rostral. The intercostal nerves were stimulated by a Medelec MS 92 stimulator with a supramaximal rectangular pulse of $0.5 \mathrm{~ms}$ duration. The distal points of stimulation were about $6 \mathrm{~cm}$ behind the costal margin and the proximal sites were just lateral to the paraspinal muscles in the same space. The ground electrode was a metallic plate of $2.5 \times 5.0 \mathrm{~cm}$ placed between the stimulating and recording electrodes. Recording of motor response was done by using $5 \mathrm{~mm}$ tin-disc surface electrodes placed over the rectus abdominis of the same side. Ideal recording sites for the individual lower five intercostal nerves were determined by placement of the electrodes at multiple sites. For this, arbitrary transverse lines were drawn at the xiphoid process, umbilicus, at one third intervals between them, and at a mid point between the pubic symphysis and umbilicus. Recording points were chosen at the middle of the rectus abdominis, one on each of these lines and three more at equal distances between each of the two lines (fig 1). At the points where best results were obtained four more recordings were made in the transverse plane, two each on either side of the middle one (fig 2). The reference electrode was placed $5 \mathrm{~cm}$ rostral to the active recording electrode except in the case of the 7th intercostal nerves where it was kept only $3 \mathrm{~cm}$ above the active electrode. Two to $10 \mathrm{KHz}$ filters were used. The latencies were measured at a $5 \mathrm{~ms} / \mathrm{div}$ sweep speed and $100 \mu \mathrm{V} /$ div voltage gain. For amplitude and duration measurement $10 \mathrm{~ms} /$ div sweep speed and a suitable voltage gain were used. The distance between the two points of stimulation was measured with a calliper. Nerve conduction velocities were calculated. All the recordings were on the right side. For comparison left sided nerves were also tested in eight subjects. Attempts were made to record the $\mathrm{H}$-reflex with the use of averaging technique at sub-threshold stimulation and minimal contraction of rectus abdominis. $F$ waves were also sought, by using supramaximal stimulation. 


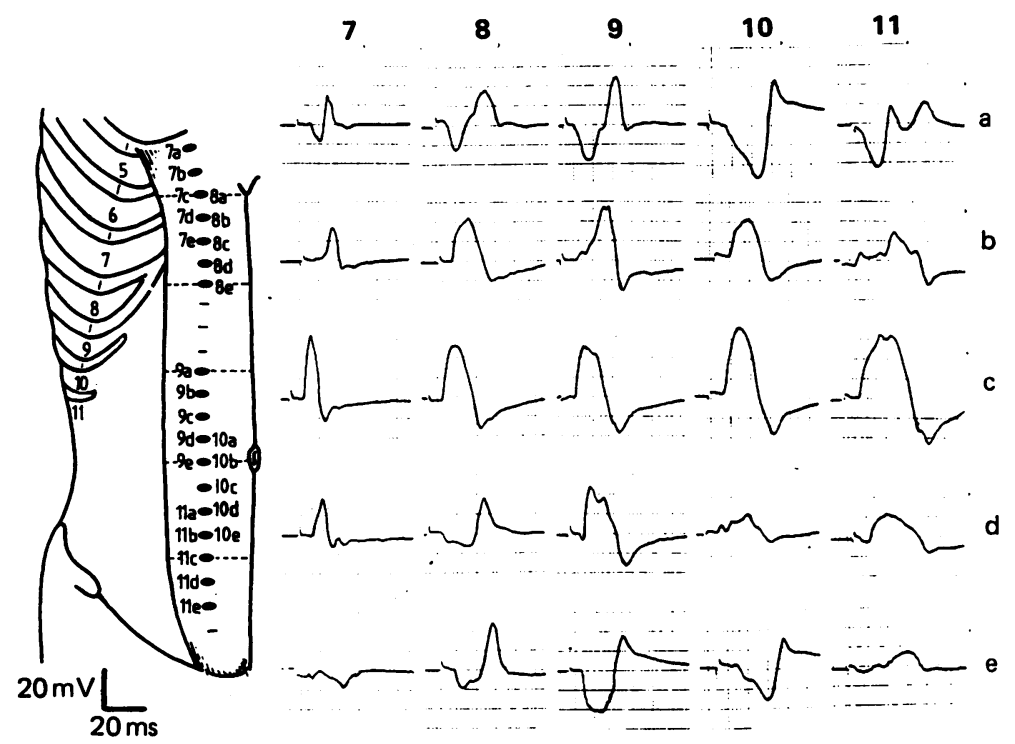

Fig 1 The CMAP recorded at different sites in the vertical plane at the middle of the width of rectus abdominis muscle in one of the subjects. The best recording sites for individual intercostal nerves are shown in the column $C$ with 4 more recordings, 2 above and 2 below the best one.

\section{Results}

Seventh to 11 th intercostal nerves could be stimulated with a constant voltage stimulator at a stimulus threshold of 70-125 V, the threshold being higher for proximal sites. Supramaximal stimulation was achieved at $125-175 \mathrm{~V}$ with proximal stimulation at about $40 \%$ higher voltage than that at distal sites. Specific motor points could be located for all the nerves in all the subjects by using multiple recording

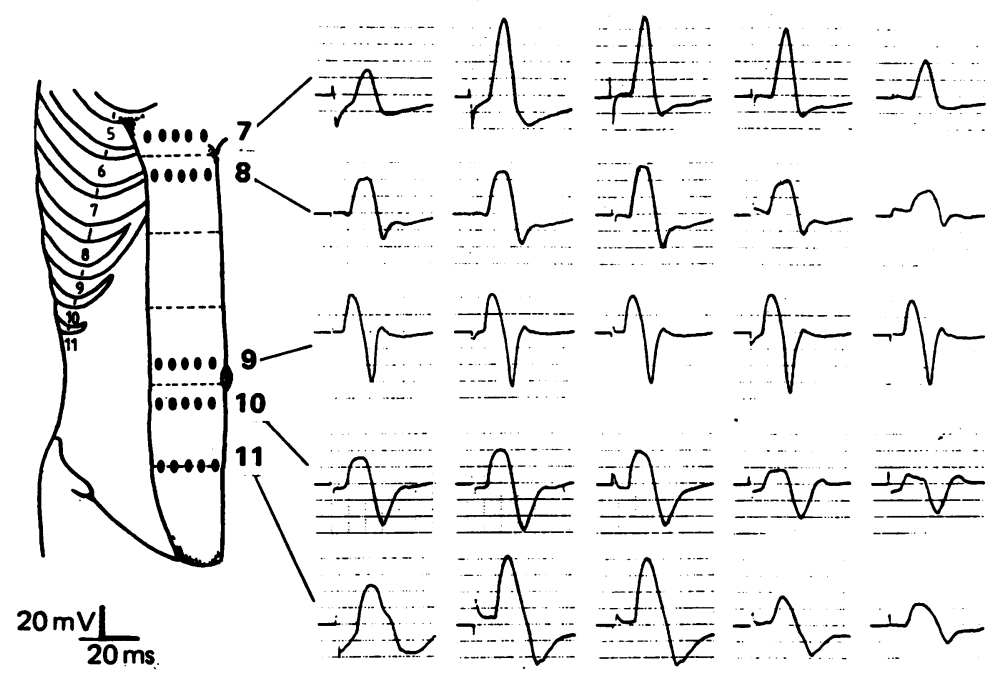

Fig 2 The CMAP recorded at different sites in the transverse plane of the rectus abdominis muscle at the level of best vertical recording site. For each nerve, recording at the middle of the widih of the muscle shows best response. 
Table Normal values (mean (SD)) of the intercostal nerve conductions derived from 30 healthy individuals, 14-52 years of age with no apparent disease of the peripheral nerves

\begin{tabular}{lllll}
\hline $\begin{array}{l}\text { Intercostal } \\
\text { nerves }\end{array}$ & $\begin{array}{l}\text { Distal } \\
\text { latency* } \\
m s\end{array}$ & $\begin{array}{l}\text { CMAP } \\
\text { amplitude } \\
m V\end{array}$ & $\begin{array}{l}\text { CMAP } \\
\text { duration } \\
m s\end{array}$ & $\begin{array}{l}\text { Conduction } \\
\text { velocity } \\
m / s\end{array}$ \\
\hline 7 th & $3.51(0.67)$ & $5.56(2.42)$ & $14.46(2.71)$ & $75.07(6.28)$ \\
8th & $3.66(0.48)$ & $4.56(2.19)$ & $16.40(3.46)$ & $74.87(5.95)$ \\
9 th & $3.96(0.31)$ & $2.80(1.54)$ & $18.43(3.56)$ & $75.52(6.37)$ \\
10 th & $4.56(0.65)$ & $2.40(1.48)$ & $19.83(3.63)$ & $74.78(6.07)$ \\
11 th & $4.98(0.61)$ & $2.60(1.37)$ & $21.49(4.20)$ & $71.67(7.43)$ \\
\hline
\end{tabular}

*With stimulation $6 \mathrm{~cm}$ behind the subcostal margin.

$\dagger$ As measured from baseline to negative peak after the distal stimulation.

sites (fig 1). At all these points, the initial major deflection of CMAP was negative and the amplitude was maximum. The CMAP was usually a negativepositive biphasic potential with fairly sharp onset point. A small variation was observed in the motor points in different individuals. The most consistent recording sites in the majority of the subjects were: 7th intercostal nerves 0 to $1 \mathrm{~cm}$ above the level of xiphoid process; 8 th intercostal nerves, $1-3 \mathrm{~cm}$ below the level of xiphoid process; 9th intercostal nerves, $2-5 \mathrm{~cm}$ above the level of umbilicus; 10th intercostal nerves, $1 \mathrm{~cm}$ above to $4 \mathrm{~cm}$ below the level of umbilicus and 11 th intercostal nerves, mid way between umbilicus and public symphysis. Only a little adjustment was required in the longitudinal plane within the aforementioned range. In the transverse plane the best CMAP was obtained at the middle of the width of rectus abdominis (fig 2). The distal latencies, amplitudes and durations of CMAP and the conduction velocities of different intercostal nerves are shown in the table. No significant difference was observed in the mean velocities of different intercostal nerves. Comparison between right and left intercostal nerves also did not show a significant difference in conduction velocities but the amplitudes showed marked variability. On repeating the conduction studies on two different occasions in five subjects, the results were reproducible. The $F$ wave could be elicited superimposed on the repolarisation wave whenever the duration of CMAP was small. The $\mathrm{H}$ reflex could not be obtained in any of the subjects.

\section{Discussion}

The earlier physiological studies by Campbell ${ }^{67}$ and Taylor ${ }^{8}$ have shown that the lower intercostal muscles and the abdominal muscles play a more significant role in respiration than the upper intercostal muscles. We therefore concentrated on the conduction study of lower five intercostal nerves and found the rectus abdominis muscle to be the ideal recording site. The rectus abdominis is innervated by the lower six or seven thoracic nerves. The seventh to 11 th intercostal nerves supply the muscle invariably. We, therefore, studied only the 7th to 11 th intercostal nerves and as expected, CMAP could be obtained upon stimulation of all of these in all individuals.

As there are no available details of the motor points for different intercostal nerves in the rectus abdominis, multiple recording sites were required to locate these points. The exact points could be identified for all the nerves. These were fairly consistent in different individuals on both the sides and in the same individual on different occasions. If anatomical landmarks for motor points were recorded, serial studies may be conducted with reliable results.

Certain precautions regarding the patient's position, nerve stimulation and distance measurements were found to be necessary. Change of posture between distal and proximal stimulation made a little but significant difference in the distance between the two points of stimulation, thereby altering the calculation of the conduction velocity. To stimulate both proximal and the distal points without a change in posture, a semiprone position preferably with a pillow under the chest was satisfactory, the latter resulting in prominence and widening of the intercostal spaces. Similarly, putting the higher arm in front of the face proved very useful as it moved the scapula and the attached muscles forwards and upwards allowing easy access to the 7th and 8th intercostal spaces for proximal stimulation. Relaxation was required to ensure measurements in a neutral chest position. A gentle firm pressure deep into the intercostal spaces was mandatory to obtain a sharp take-off of CMAP. The distal point of stimulation was $6 \mathrm{~cm}$ behind the costal margin. Anterior to that, the nerves were too deep for proper surface stimulation. Proximally all intercostal nerves could be stimulated lateral to the paraspinal muscles. The morphology of CMAP was identical from distal and proximal sites of stimulation and this feature was used to identify the intercostal spaces in difficult circumstances, such as obesity. Owing to the deep situation of the nerves, $0.5 \mathrm{~ms}$ stimulus duration was found to be better than the usual $0.2 \mathrm{~ms}$. In highly obese or muscular persons one may require even higher stimulus duration to obtain a proper CMAP. Since the intercostal nerves have a circular course and the measurement between the two points of stimulation is done far outside the inner "arc", one is bound to get falsely high velocities unless a calliper is used for the measurement. Sometimes, owing to a small 12 th rib, enough distance could not be obtained to measure the velocity of the 11 th intercostal nerves. In these cases, distal stimulation was carried out by putting the stimulating electrode over the abdominal wall just in front of the 11th intercostal spaces. The CMAP was better in amplitude, con- 
figuration and initial take off when the reference electrode was placed $5 \mathrm{~cm}$ above the active electrode. Therefore, the possibility of the reference electrode also playing an active role, cannot be ruled out.

Inconsistency in obtaining $\mathbf{F}$ waves was probably due to the short nerve length proximal to the site of stimulation and wide CMAP. An attempt was made to shorten CMAP by using a needle recording electrode but without success.

The technique was also applied to five cases of Gullian Barré syndrome including the two with respiratory paralysis. Among the latter, one had absent CMAP and another showed reduced conduction from all the intercostal nerves. Among the other three cases, one showed abnormal conduction in right 7 th and left 9th intercostal nerves while the other two cases showed normal intercostal nerve conduction.

This technique can be applied to measure nerve conduction velocities of the lower five intercostal nerves with fairly reliable results. In the absence of consistent late responses, its practical utility appears to be restricted to evaluation of various truncal neuropathies, truncal involvement in generalised neuropathies, nerve injuries due to trauma or surgery and in the evaluation of post laparotomy hernias.

The utility of intercostal nerve conduction in the evaluation of respiratory paralysis may be great because the movements of the diaphragm though responsible for about $75 \%$ of tidal respiration, are not essential for respiration. Even bilateral diaphragmatic palsy, in the absence of serious lung disease, may sometimes cause no serious disability. ${ }^{9}$ Therefore, in cases of selective involvement of the respiratory muscles leading to respiratory paralysis without limb weakness, as sometimes seen in acute inflammatory demyelinating polyradiculoneuropathy of autoimmune origin, ${ }^{1011}$ intercostal nerve conduction study may play an important role.
This work was supported by a grant funded by the Council for Scientific and Industrial Research, New Delhi, India.

\section{References}

1 Sun SF, Streib EW. Diabetic thoracoabdominal neuropathy: Clinical and electrophysiological features. Ann Neurol 1981;9:75-9.

2 Streib EW, Sun SF, Paustian FF, et al. Diabetic thoracic radiculopathy: electrodiagnostic study. Muscle Nerve 1986;9:548-53.

3 Boulton AMJ, Angnus E, Ayyar DR, et al. Diabetic thoracic polyradiculopathy presenting as abdominal swelling. Br Med J 1984;289:798-9.

4 Thomas JE, Howard SM Jr. Segmental Zoster paralysis -a disease profile. Neurology 1972;22:459-66.

5 Glantz RH, Ristanovic RK. Abdominal muscle paralysis from herpes zoster. J Neurol Neurosurg Psychiatry 1988;52:885-6.

6 Campbell EJM. An electromyographic examination of the role of the intercostal muscles in breathing in man. J Physiol (Lond) 1955;129:12-26.

7 Campbell EJM, Green JH. The expiratory function of the abdominal muscles in man. An electromyographic study. J Physiol (Lond) 1953;120:409-18.

8 Taylor A. The contribution of intercostal muscles to the effect of respiration in man. J Physiol (Lond) 1960; 151:390-402.

9 Keele AC, Neil E. Mechanism of breathing. In: Samson Wright's Applied Physiology. Oxford: Oxford University Press, 1965:158-9.

10 Schmalstieg EJ, Peters BH, Schochat SS, Findlay SR. Neuropathy presenting as prolonged dyspnoea - a case report and review of literature. Arch Neurol 1977; 34:473-6.

11 Goldstein RL, Hyde RW, Lapham LW, et al. Peripheral neuropathy presenting with respiratory insufficiency as the primary complaint. Problem of recognizing alveolar hypoventillation due to neuromuscular disorders. Am J Med 1974;56:443-9. 